\title{
Acceptance of the Kober Medal It only gets better
}

\author{
K. Frank Austen, M.D.
}

As all of you know, the New England Journal of Medicine embargoes its presentations until the date of public release. I deeply appreciate Jeff's generous remarks. We have enjoyed a wonderful collaboration over several decades.

My choice to study inflammation, an area that was not popular, was guided by wonderful early mentors. These individuals included Walter Bauer at the Massachusetts General Hospital (MGH), who emphasized the importance of acknowledging what one did not know when providing patient care, specifically to patients with inflammatory arthritis; Elmer Becker at the Walter Reed Army Institute of Research, who directed me to the study of the biologic consequences rather than the exquisite specificity of an immune reaction and arranged for me to move on to John Humphrey's program at Mill Hill, England; Walter Brocklehurst, who taught me how to generate and bioassay slow-reacting substance of anaphylaxis (cysteinyl leukotrienes) using smoothmuscle preparations; Manfred Mayer of Johns Hopkins, who emphasized that serious research was characterized by "deep mining," not "surface mining"; and Bob Ebert, who as Dean of Harvard Medical School conceived my move from the MGH to the Robert B. Brigham Hospital and the complex now known as the Brigham and Women's Hospital to expand the effort in studying the inflammatory response.

The composite outcome of their guidance has been my 45 years of focused research in inflammation, directed to the alternative complement pathway, the cysteinyl leukotrienes, and the mast cell. The inflammatory process is now dignified by the term "innate immunity." Irrespective of nomenclature, inflammation precedes and primes an adaptive immune response and then follows that response to provide biologic meaning. Inflammation/pattern recognition is the big arena within which

This article is adapted from a presentation at the ASCI/AAP Joint Meeting, April 16-18, 2004, in Chicago, Illinois, USA.

Citation for this article: J. Clin. Invest. 114:1177 (2004). doi:10.1172/JCI200423465. adaptive immunity operates as one of the controls. Importantly, there is increasing evidence of counter-regulatory or attenuating signals for both the innate and adaptive components of immunity. "Turn-off" mechanisms are as important as activation pathways, regardless of specificity; without them, we would be consumed by our inflammatory responses.

My submitted title for my response to receiving the Kober Medal from the Association of American Physicians was It only gets better with age and experience. Experience and focus provide a context to which new technology is easily and strategically added. Only this year, 2004, after studying slow-reacting substance of anaphylaxis for 45 years did we unexpectedly find a new set of functions. Through a second receptor, cysteinyl leukotrienes have a role in chronic inflammation. By chronic inflammation, I mean the accumulation of macrophages and fibroblasts with the laying down of extracellular matrix such as collagen. Although the acute effects of the cysteinyl leukotrienes were revealed in pharmacologic studies of animals and humans, the role of these mediators in chronic inflammation was uncovered only through transgenic approaches in which inflammation was induced in strains with targeted disruption of individual receptors. Truly, it only gets better.

Like many in academic medicine, I have benefited mightily by "reverse mentoring" from pre- and postdoctoral fellows. To choose to study inflammation rather than lymphocyte biology, these trainees were inherently a risk-taking group. They made certain that we explored the primitive area of host inflammatory responses with state-of-the-art technology. Happily, many were physicians who found it easy to relate this subject to their clinical work, which, in turn, reinforced and modulated our laboratory program.

Another benefit of my career in biomedical science has been my interactions with other investigators in laboratories around the world. Together, we have reached shared goals that could not have been achieved in a single laboratory or through a single discipline. The unraveling of the cysteinyl leukotriene pathway and its functions derived from interdisciplinary research involving the biochemistry of Bengt Samuelsson in Sweden, the synthetic chemistry of E.J. Corey at Harvard, the physiologic expertise of Jeff Drazen, and numerous others. These friendships between laboratories and between families continue independently of any ongoing shared science.

Finally, I am blessed with a most joyful and tolerant spouse, Joycelyn, who allowed my avocation and the accompanying need to live in five-year funding cycles. Similarly, our four children and now our son- and daughters-in-law share Joycelyn's immunity to my shortcomings and amusement when I attempt to direct their activities in any fashion. Joycelyn is both the source and the glue of the wonderful family that I appreciate so very much.

I thank the Association of American Physicians for allowing me to share in the honor of this medal with those who have preceded and those who will follow.

Address correspondence to: K. Frank Austen, Smith Building, Room 638, One Jimmy Fund Way, Brigham \& Women's Hospital, Boston, Massachusetts 02115. E-mail: Fausten@rics.bwh.harvard.edu. 